\title{
Interactions of Bacterial Quorum Sensing Signals with Model Lipid Membranes: Influence of Acyl Tail Structure on Multi-Scale Response
}

\author{
Curran G. Gahan, ${ }^{1}$ Reid C. Van Lehn, ${ }^{1, *}$ Helen E. Blackwell, ${ }^{2, *}$ and David M. Lynn ${ }^{1,2, *}$
}

${ }^{1}$ Department of Chemical and Biological Engineering, University of Wisconsin-Madison, 1415 Engineering Dr., Madison, WI 53706, USA; ${ }^{2}$ Department of Chemistry, University of WisconsinMadison, 1101 University Ave., Madison, WI 53706, USA; Email: (R.C.V.) vanlehn@wisc.edu; (H.E.B.)blackwell@chem.wisc.edu (D.M.L.)dlynn@engr.wisc.edu

\section{SUPPORTING INFORMATION}

\section{Supplementary Videos.}

Video S1: Representative time-lapse fluorescence microscopy video showing the formation of a SLB by bicelle fusion. In this video, a suspension of bicelles at $0.031 \mathrm{mM}$ was introduced to a glass substrate in a flow cell and allowed to adsorb to the surface over time, eventually fusing to form a continuous, fluid SLB. The scale bar represents $30 \mu \mathrm{m}$.

Video S2: Representative time-lapse fluorescence microscopy experiment characterizing the influence of $50 \mathrm{uM} 3$-oxo-C10-AHL on a SLB. In this video, a $50 \mu \mathrm{M}$ 3-oxo-C10-AHL solution was added to a fluorescently labeled DOPC SLB, leading to the formation of extended tubules and hemispherical caps on the surface of the bilayer. Most of the tubules persist on the surface of the bilayer over the time of the experiment. The scale bar represents $30 \mu \mathrm{m}$.

Video S3: Representative time-lapse fluorescence microscopy experiment characterizing the influence of $50 \mathrm{uM} \mathrm{C10-AHL}$ on a SLB. In this video, a $50 \mu \mathrm{M} \mathrm{C10-AHL} \mathrm{solution} \mathrm{was} \mathrm{added} \mathrm{to}$ a fluorescently labeled DOPC SLB, leading generally to the formation of hemispherical caps on the surface of the bilayer. The scale bar represents $30 \mu \mathrm{m}$.

Video S4: Representative time-lapse fluorescence microscopy experiment characterizing the influence of $50 \mathrm{uM} 3$-oxo-C12-AHL on a SLB. In this video, a $50 \mu \mathrm{M}$ 3-oxo-C12-AHL solution was added to a fluorescently labeled DOPC SLB, leading to the formation of extended tubules, some of which collapse over time into hemispherical caps on the surface of the bilayer. Some of the tubules persist on the surface of the bilayer over the time of the experiment. The scale bar represents $30 \mu \mathrm{m}$.

Video S5: Representative time-lapse fluorescence microscopy experiment characterizing the influence of $50 \mathrm{uM} \mathrm{C12-AHL}$ on a SLB. In this video, a $50 \mu \mathrm{M}$ C12-AHL solution was added to a fluorescently labeled DOPC SLB, leading generally to the formation of hemispherical caps on the surface of the bilayer. Many of these hemispherical caps then retract in size and new caps form on the surface of the bilayer. The scale bar represents $30 \mu \mathrm{m}$. 
Video S6: Representative time-lapse fluorescence microscopy experiment characterizing the influence of $50 \mathrm{uM} 3$-oxo-C14-AHL on a SLB. In this video, a $50 \mu \mathrm{M}$ 3-oxo-C14-AHL solution was added to a fluorescently labeled DOPC SLB, leading to the formation of extended tubules and hemispherical caps on the surface of the bilayer. Some of these tubules collapse into caps over time, while others persist on the surface of the bilayer over the timescale of the experiment. The scale bar represents $30 \mu \mathrm{m}$.

Video S7: Representative time-lapse fluorescence microscopy experiment characterizing the influence of $50 \mathrm{uM} \mathrm{C14-AHL}$ on a SLB. In this video, a $50 \mu \mathrm{M}$ C14-AHL solution was added to a fluorescently labeled DOPC SLB, leading generally to the formation of small hemispherical caps on the surface of the bilayer. The scale bar represents $30 \mu \mathrm{m}$.

Video S8: Representative time-lapse fluorescence microscopy experiment characterizing the influence of $50 \mathrm{uM} 3$-oxo-C16-AHL on a SLB. In this video, a $50 \mu \mathrm{M}$ 3-oxo-C16-AHL solution was added to a fluorescently labeled DOPC SLB, leading to the formation of extended tubules and hemispherical caps on the surface of the bilayer. Some of these tubules collapse into caps over time, while others persist on the surface of the bilayer over the timescale of the experiment. The scale bar represents $30 \mu \mathrm{m}$.

Video S9: Representative time-lapse fluorescence microscopy experiment characterizing the influence of $50 \mathrm{uM} \mathrm{C16-AHL}$ on a SLB. In this video, a $50 \mu \mathrm{M}$ C16-AHL solution was added to a fluorescently labeled DOPC SLB, leading generally to the formation of small hemispherical caps on the surface of the bilayer. The scale bar represents $30 \mu \mathrm{m}$.

Note: Each individual frame of the above videos was acquired five seconds apart, and six images are shown per second. Therefore, all the videos above are sped up 30x from "real time". 
NMR Quantification of Phospholipids. To determine the concentration of phospholipids in stock solutions, quantitative ${ }^{31} \mathrm{P}\left[{ }^{1} \mathrm{H}\right]$ NMR spectroscopy was performed in $90 \%$ $\mathrm{H}_{2} \mathrm{O}$ and $10 \% \mathrm{D}_{2} \mathrm{O}$ using a Bruker Avance- 400 spectrometer with a $\mathrm{BBFO}$ probe. A relaxation delay (D1) of $11 \mathrm{sec}$ was determined by inversion-recovery pulse sequence experiments. A phospholipid vesicle sample $(890 \mu \mathrm{L}$ in water) was mixed with $10 \mu \mathrm{L}$ of Triton X-100 or rhamnolipid solution (to fully lyse the vesicles), and $100 \mu \mathrm{L}$ of a triethylphosphine oxide (TPO) solution (100 $\mathrm{mM}$ in $\left.\mathrm{D}_{2} \mathrm{O}\right)$ was added as an internal standard. Integrations for phospholipid peaks were performed relative to the phosphorus peak of TPO ( $\delta \sim 65 \mathrm{ppm})$. NMR acquisition parameters were as follows: PULPROG (pulse program) $=$ zgig30, D1 (relaxation delay) $=11 \mathrm{~s}$, $\mathrm{SW}($ sweep width $)=405 \mathrm{ppm}, \mathrm{O} 2 \mathrm{P}($ transmitter frequency of $\mathrm{f} 2$ channel $)=3.75 \mathrm{ppm}$, NS $($ number of scans $)=64$, DS (number of dummy scans $)=4, \mathrm{LB}$ (line broadening factor) $=1$.

Determination of CAC by Light Scattering. Figure S1 shows the static light scattering data used to determine the critical aggregation concentrations (CACs) listed in Table 1 of the main text. Linear regressions of scattered light intensity were performed separately for the points above and below the concentration at which the scattered light intensity of AHL solutions began to increase at increasing AHL concentrations. The CAC for each AHL was defined as the concentration corresponding to the intersection of these two regression lines. The CACs reported in Table 1 are the averages and standard deviations of the three samples shown as three separate colors in Figure S1. For some AHLs with longer tails, linear regressions of acquired data could not be performed with certainty. In these cases, we report estimated upper bounds of CAC values corresponding to the lowest concentration that was co-linear with values obtained at higher concentrations. Those reported values also corresponded to the lowest concentrations tested that exhibited clear deviations from background levels of scattered light intensity. For these longertailed AHLs, the values reported below thus represent an estimated upper bound of the CACs, and the actual CACs are likely lower than those values. 

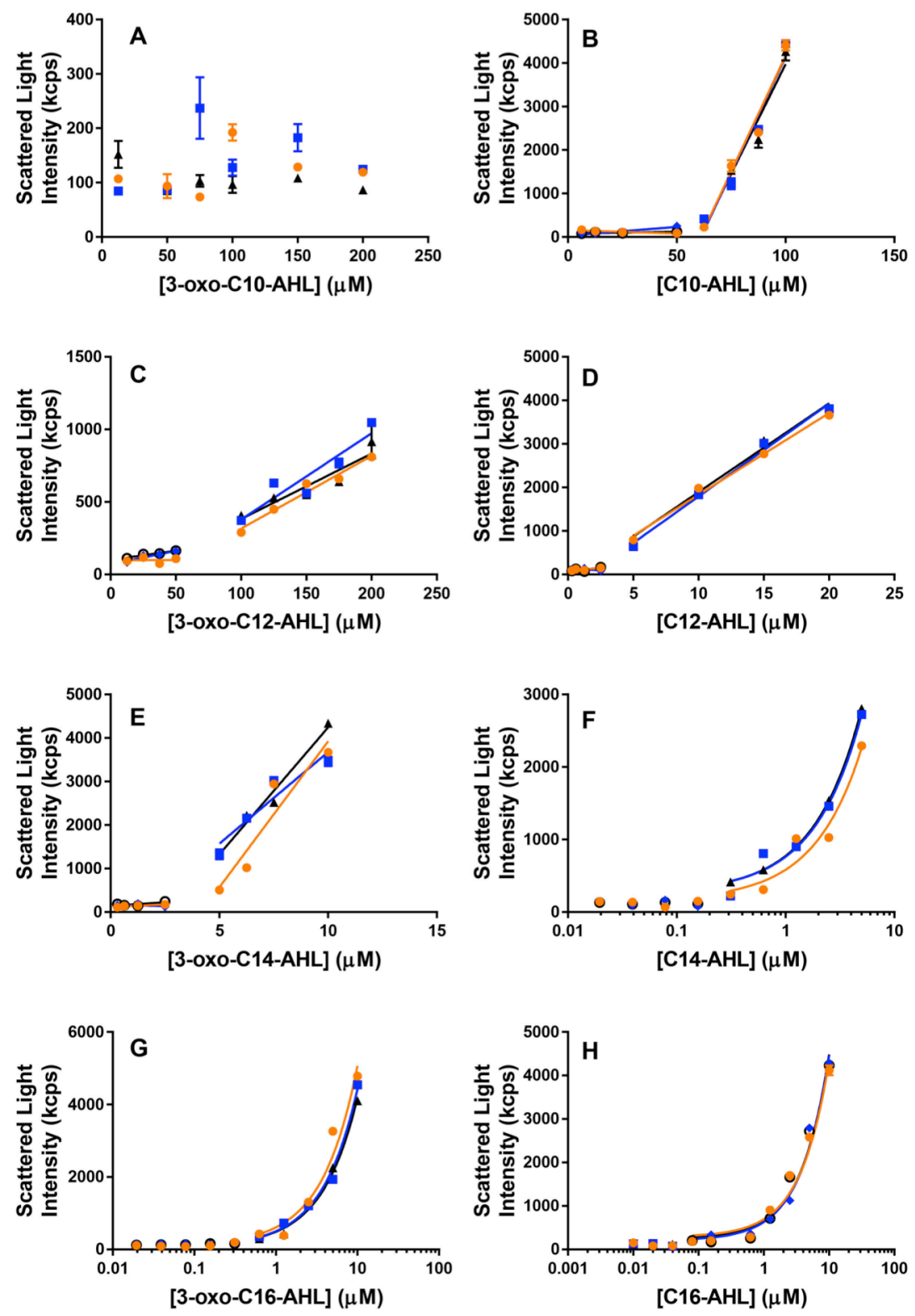

Figure S1: Determination of the critical aggregation concentrations (CACs) of the AHLs shown in Table 1 using static light scattering in buffer containing 1\% DMSO. All points represent the average and standard deviation of three independent measurements of individual solutions of AHLs. Black, orange, and blue data points show results obtained for three sets of independently prepared samples. Note that AHL concentration in some panels is plotted on a $\log$ scale. The plots show the scattered light intensity of a $532 \mathrm{~nm}$ incident laser, detected at $173^{\circ}$, and at various concentrations of (A) 3-oxo-C10-AHL, (B) C10-AHL, (C) 3-oxo-C12AHL, (D) C12-AHL, (E) 3-oxo-C14-AHL, (F) C14-AHL, (G) 3-oxo-C16-AHL, and (H) C16AHL. 

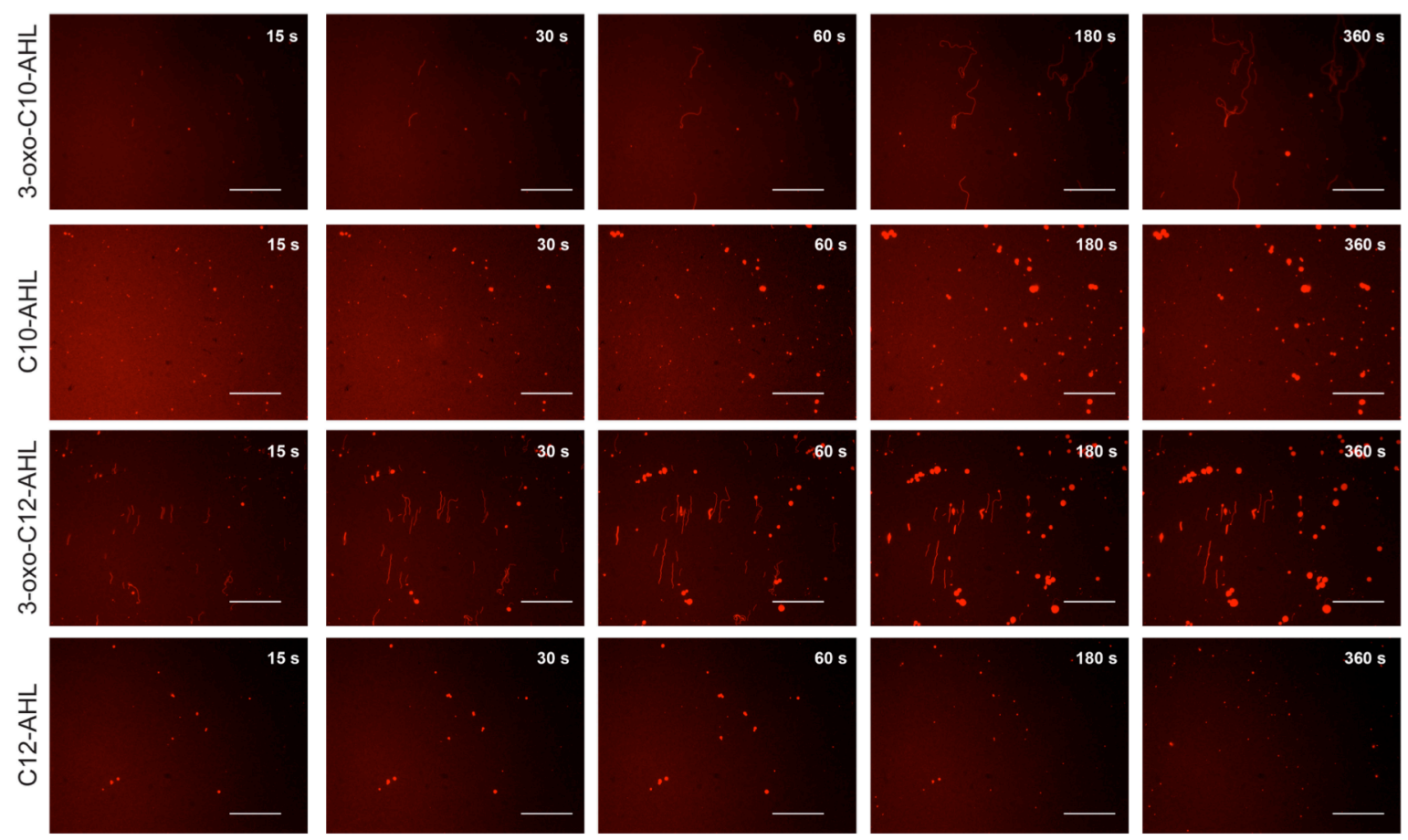

Figure S2A: Fluorescence micrographs showing the restructuring in DOPC SLBs after the introduction of $50 \mu \mathrm{M}$ solutions of (top to bottom) 3-oxo-C10-AHL, C10-AHL, 3-oxo-C12AHL, or C12-AHL over time. The images shown were acquired at various time points indicated in the top-right corner of the images after the first membrane restructuring event was observed. The 360-second images shown here are the same as those shown in Figure 2 of the main text. The AHL examined in each experiment is shown at the left of each row of images. The direction of flow in all the images was from the bottom to the top of the panel. Scale bars represent $30 \mu \mathrm{m}$. Some features in these images are easier to observe and discern when the images are enlarged and can also be observed in the supplemental videos. 

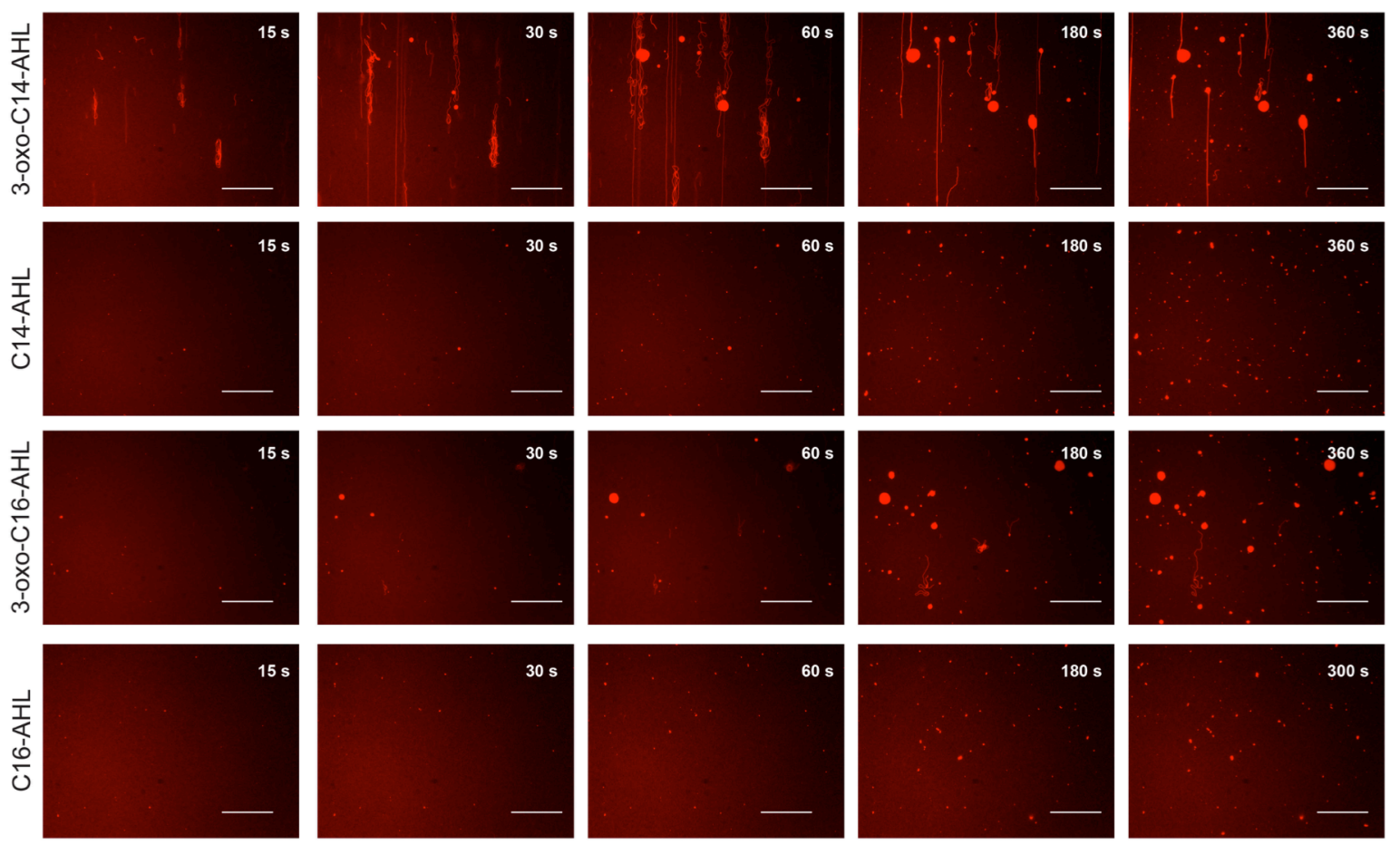

Figure S2B: Fluorescence micrographs showing the restructuring in DOPC SLBs after the introduction of $50 \mu \mathrm{M}$ solutions of (top to bottom) 3-oxo-C14-AHL, C14-AHL, 3-oxo-C16AHL, or C16-AHL over time. The images shown were acquired at various time points indicated in the top-right corner of the images after the first membrane restructuring event was observed. The 300-second or 360-second images shown here are the same as those shown in Figure 2 of the main text. The AHL examined in each experiment is shown at the left of each row of images. The direction of flow in all the images was from the bottom to the top of the panel. Scale bars represent $30 \mu \mathrm{m}$. Some features in these images are easier to observe and discern when the images are enlarged and can also be observed in the supplemental videos. 

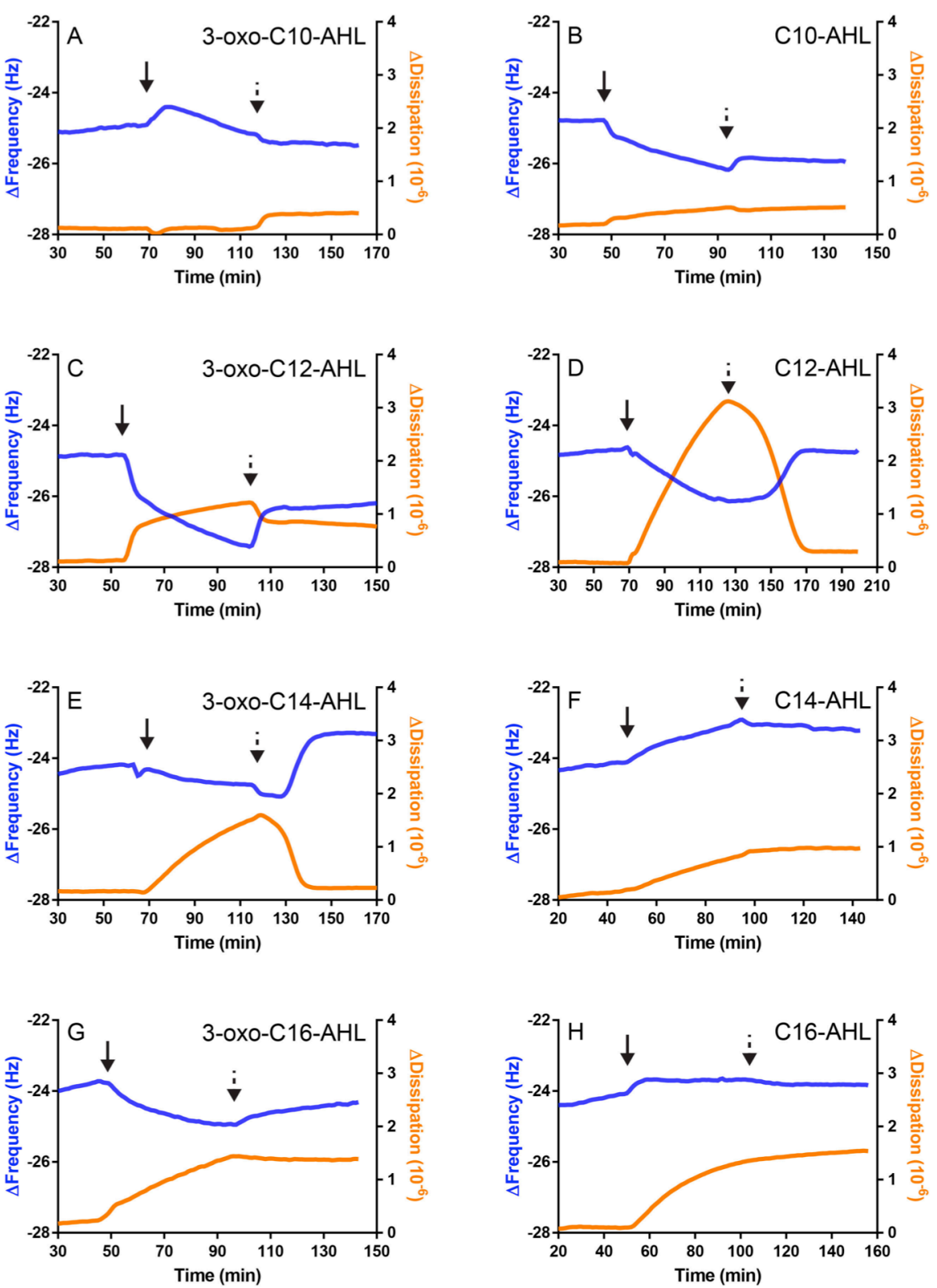

Figure S3: Representative QCM-D measurements of large-scale bilayer reformations in DOPC SLBs initiated by the introduction of $50 \mu \mathrm{M}$ solutions of (A) 3-oxo-C10-AHL, (B) C10-AHL, (C) 3-oxo-C12-AHL, (D) C12-AHL, (E) 3-oxo-C14-AHL, (F) C14-AHL, (G) 3oxo-C16-AHL, and $(\mathrm{H}) \mathrm{C} 16-\mathrm{AHL}$. The changes in frequency ( $\Delta$ Frequency, blue lines) and dissipation ( $\Delta$ Dissipation, orange lines) shown are representative of three or four independent experiments. The initial measurement values $(\mathrm{t}=0 \mathrm{~min})$ of these experiments correspond to a DOPC SLB on an $\mathrm{SiO}_{2}$ surface. AHL solutions were introduced to the bilayer at the time indicated by the solid arrow and a buffer wash was introduced at the time indicated by the dashed arrow. 